\title{
Wavy Aspects in a Precessed Cylindrical Open Channel
}

\author{
Hajar Alshoufi \\ Faculty of Civil Engineering, Budapest University of Technology and Economics, Budapest, Hungary
}

\section{Email address:}

hajar.alashoufi@emk.bme.hu

\section{To cite this article:}

Hajar Alshoufi. Wavy Aspects in a Precessed Cylindrical Open Channel. International Journal of Fluid Mechanics \& Thermal Sciences. Vol. 7, No. 3, 2021, pp. 22-26. doi: 10.11648/j.ijfmts.20210703.11

Received: October 25, 2021; Accepted: November 12, 2021; Published: November 23, 2021

\begin{abstract}
In this letter some experimental and theoretical results from the flow in open cylindrical channel under precession conditions are reported. This includes the linear solutions of velocity field based on real experimental observations, and the nonlinear solution where a new weakly nonlinear KdV model equation is derived and solved numerically, this was compared with the real single Kelvin mode of the problem that appears in a symmetrical form with constant speed. The paper also shades the light on the instability features, mainly the wave breaking of single Kelvin mode, that is considered the resonance case of the problem, a new instability diagram in terms of Strouhal and Reynolds numbers is presented for the different cases reported from the experiment. The measurements of the mean azimuthal flow have been carried out using particle tracking procedure that enabled us finally to extract equation fit connects between the control parameters of the problem (which are the water volume, the angle of tilt and rotation rate) and the drift velocity the results pours in favor of the ADV measurements extracted in the previous work, and show that the mean flow increases by increasing both the tilt and the rotation rate where the instability features in the system appear at earlier levels, while increasing the water volume needs more energy to get the flow instability.
\end{abstract}

Keywords: Precession, Open Channel Flow, KdV Equation

\section{Introduction}

When precession effect exists in the background of the flow, inertial oscillations are excited and appeared as natural results in such systems, the detuning Coriolis force effect adds additional momentum into the water body that causes those inertial oscillations as long as the forcing frequency smaller than twice the base rotation rate [1]. This will lead to ill-posed hyperbolic boundary value problem that gives a dense set of eigenmodes that were first introduced in the previous study [3], each mode accords with eigenfrequency. When the forcing frequency matches the natural one predicted by the modes, we call it resonance situation, for the open channel case [3], the forced mode is the single Kelvin one and, in this case, the wavelength matches the outer periphery of the cylinder. In case of closed cylinders, many studies $[4,5]$ showed that resonance depends on the aspect ratio between the cylinder height and its radius. Precession applications are varied, in planetary cores and stars [6,7], aeronautics [8,9], also in spin-stabilized artillery projectiles [10]. A common interesting idea that accompanies such waves is the instability phenomenon; it was revealed that those inertial waves are generically unstable and, in many cases, catastrophically, so quickly causing the whole flow to collapse to small-scale disorder [11]. Similar to the waves studied in closed containers (spheres, boxes, cylinders), the investigation of open flows focused on a cylinder that is filled partially under precession conditions $[12,13]$, or lately a new case study in open channel by the present author [3], where the cavity is replaced with two concentric cylinders with radii $r_{\text {max }}, r_{\text {min }}$ respectively for the outer and inner cylinders, form an annulus that tilts and rotates with width difference $r=r_{\text {max }}-r_{\text {min }}$. The instability features in such case noticed in the form of degeneration of single Kelvin mode into dispersive form or wave breaking and turbulent motion was observed. Full details of the experimental apparatus and procedure are in the previous work [3]. The cylinders are mounted on the lab floor (the inertial frame of reference) using metal support. The cylinders rotate about their common axis $(O, \hat{k})$ with precession rate $\Omega_{2}=\tau \Omega$, where $\tau$ the slope of the upper table which is the measure of tilt angle $\tau=$ $\tan (\psi)$. The lower table is able to rotate about an axis $(O, z)$ with rotation rate $\Omega$. The angle between the local axis $\hat{k}$ and the inertial one $z$ is the angle of tilt $\psi$. The cylinders are filled with water of different volumes, the inner cylinder has higher 
height than the outer one.

\section{Governing Equations}

The flow conditions are assumed irrotational inviscid ones, the velocity potential leads to Laplace equation for the field equation and Bernoulli equation for energy:

$$
\begin{gathered}
\frac{\phi_{\theta \theta}}{r^{2}}+\frac{\phi_{r}}{r}+\phi_{r r}+\phi_{z z}=0 \\
\phi_{t}+\frac{\phi_{\theta}^{2}}{2 r^{2}}+\frac{\phi_{r}^{2}}{2}+\frac{\phi_{z}^{2}}{2}+g \eta=g \tau \cos (\Omega t-\theta) r+O\left(\tau^{2}\right) .
\end{gathered}
$$

Where the velocity potential function is $\phi(t ; r, \theta, z)$, the spatial coordinates are $(r, \theta, z)$ in the radial, azimuthal and axial direction, respectively, $\eta(t ; r, \theta)$ the surface function, the free surface under the atmospheric conditions thus, the pressure is assumed zero $\frac{P}{\rho}=0$. The homogeneous boundary problem solution is:

$$
\begin{gathered}
\phi(t, \theta, z, r)=\frac{\Gamma}{2 \pi} \theta+\sum_{m=1}^{\infty} \sum_{\sigma=1}^{2} \tilde{\phi}_{0 m} \phi_{0 m}(z, r)+ \\
\sum_{n=1}^{\infty} \sum_{m=0}^{\infty} \sum_{\sigma=1}^{2} \tilde{\phi}_{+n m \sigma} \phi_{+n m \sigma}(t, \theta, z, r)+ \\
\sum_{n=1}^{\infty} \sum_{m=0}^{\infty} \sum_{\sigma=1}^{2} \tilde{\phi}_{-n m \sigma} \phi_{-n m \sigma}(t, \theta, z, r) .
\end{gathered}
$$

Where the first term corresponds to the primary flow $\Gamma=2 \pi r^{2} \Omega$, the second term corresponds to the secondary flow (purely standing waves) and the third and fourth terms respectively are the sum of Kelvin modes counterclockwise or clockwise. Where the velocity potential is:

$$
\phi_{n m \sigma}(t, \theta, z, r)=\varrho_{n \sigma}(\theta, t) \chi_{n m}(r) \zeta_{n m}(z) .
$$

$\varrho(\theta, t)$ the azimuthal base function, $\chi(r)$ the radial base function, and finally $\zeta(z)$ the axial base function, $n$ the integer axial wavenumber in the direction of the flow, $m$ the radial wavenumber, and $\sigma$ the azimuthal base mode which is 1 for the homogeneous case and 2 for the forced one. The amplitude of each mode is computed separately, and the resultant is given by:

$$
\tilde{\phi}_{ \pm n m \sigma}=C_{n m} \cdot v_{n m} \cdot d_{n m}
$$

Where $C_{n m}, v_{n m}, d_{n m}$ are the normalized amplitudes for each base functions for the radial, vertical and azimuthal directions respectively [3]. The final velocity vector for the problem can be taken from the derivatives of the total velocity vector $u, v, w$ in each direction (azimuthally, radially, and vertically) as follows:

$$
U=\left[\begin{array}{l}
u \\
v \\
W
\end{array}\right]=\left\{\begin{array}{l}
\frac{1}{r} \frac{\partial \phi}{\partial \theta} \\
\frac{\partial \phi}{\partial r} \\
\frac{\partial \phi}{\partial z}
\end{array}=\left[\begin{array}{l}
\hat{\rho}_{n \sigma}^{\prime}(\vartheta) \cdot \sum_{m=0}^{\infty} \chi_{n m}(r) \zeta_{n m}(z) \\
\hat{\rho}_{n \sigma}(\vartheta) \cdot \sum_{m=0}^{\infty} \chi_{n m}^{\prime}(r) \zeta_{n m}(z) \\
\hat{\rho}_{n \sigma}(\vartheta) \cdot \sum_{m=0}^{\infty} \zeta_{n m}^{\prime}(z) \chi_{n m}(r)
\end{array}\right] .\right.
$$

The simulation of the flow in the channel was carried out using Python programming language where new program is written to compute the velocity field in three dimensions and the energy for each mode separately, where the extracted solutions of the velocity field are tracked based on experimental data, the computations of the azimuthal and radial velocities are clear in Figure 1. [14]

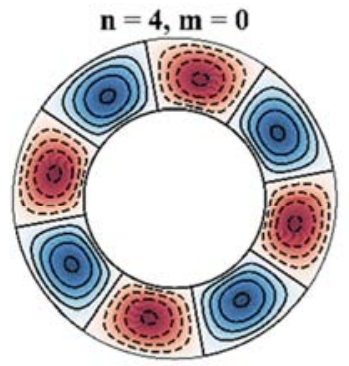

(a)

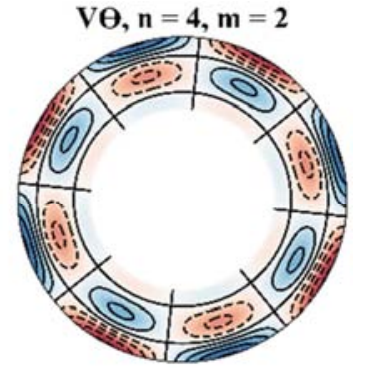

(b)
Figure 1. (a). Radial velocity field $n=4, m=0, V=8000 m l, \tau=$ 0.0333, $\Omega=4.22 \mathrm{rad} / \mathrm{s}$, (b). Azimuthal velocity field.

When taking the forcing term of Bernoulli Eq. (2), precession forces an azimuthal base mode $\sigma=2$, and axial wavenumber in the direction of the flow $n=1$, for any set of radial modes, this is the simple linear resonance case when the forcing frequency equals the natural one. The wave amplitude of this case can be given solely in terms of the radial part of velocity potential as:

$$
\eta(\theta, t, r) \cong \tau \cos (\theta-\Omega t)\left[\sum_{m=0}^{\infty} \frac{\widetilde{\Omega}_{1 m}^{2} K_{m} \chi_{1 m}(r)}{\widetilde{\Omega}_{1 m}^{2}-\Omega^{2}}\right] .
$$

Where $\tilde{\Omega}_{1 m}$ the eigenfrequency of the resonant mode, $K_{m}$ results of orthogonality between potential function and the radial direction. This amplitude in Eq. (7) becomes singularly great when the driving frequency $\Omega$ exactly equals the natural one $\tilde{\Omega}_{1 m}$, which is the resonance case of the problem, then including the nonlinear and viscous effects is needed. This matching experimentally did not occur exactly rather close to zero cases were reported, and this discrepancy was suggested by Eloy et al. [14] due to the nonlinear effect which tends to detune the frequency when the amplitude grows.

\section{Instability Observations}

Greenspan (1968) defined the instability in the fluids as follows: a given motion is unstable if the effect of any small disturbance leads to the development of either another laminar flow or a state of turbulence". The instability features in this channel are varied for instance the investigation of resonance mode showed catastrophic results as breaking occurs to the mode in different aspects, this mode accords with single azimuthal wave, sometimes the wave is flattened with high turbulence appears under the crest, or breaking into smaller waves, sometimes the crest precedes the whole body of the wave, other times disperses as it is clear in Figures 2, 3, and 4. 

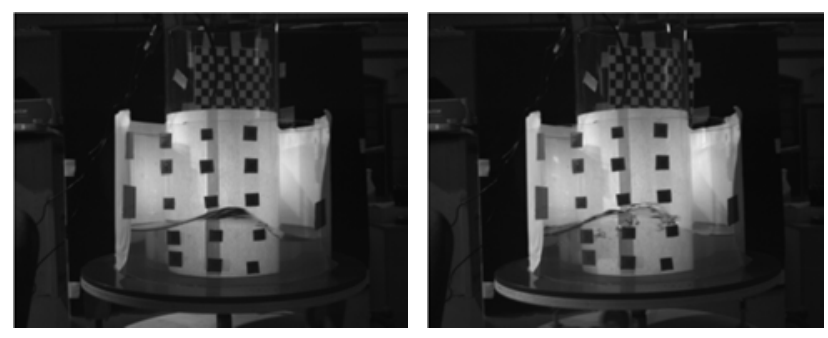

Figure 2. The mode breakes into two interlaced waves, with small scale disorder under the crest. Volume $8000 \mathrm{ml}, \Omega=6.81 \mathrm{rad} / \mathrm{s}, \tau=0.03333$.
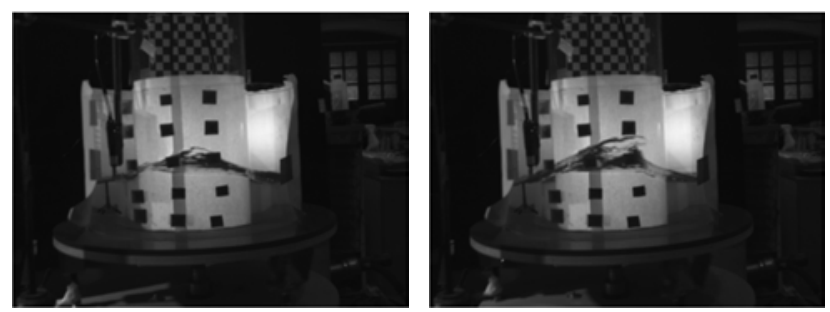

Figure 3. The wave crest proceeds the whole body. Volume 10000ml, $\Omega=6.38 \mathrm{rad} / \mathrm{s}, \tau=0.02333$.
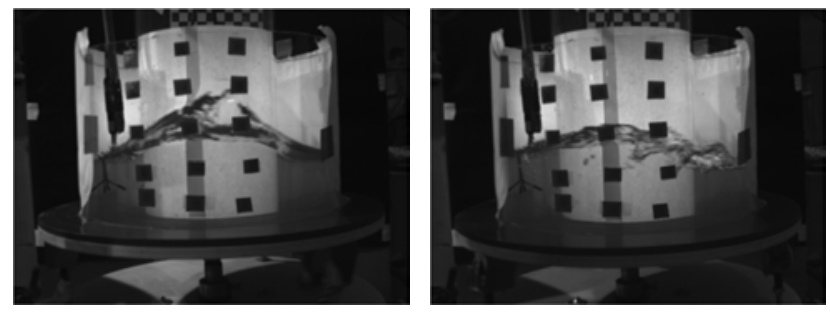

Figure 4. Flattened single Kelvin mode with turbulent agitated area after many rounds. Volume $10000 \mathrm{ml}, \Omega=6.84 \mathrm{rad} / \mathrm{s}, \tau=0.01667$.

The treatment of instability depended on the real observations using $\mathrm{CCD}$ camera that records the motion continuously, as mentioned that there are three control parameters, one of them is the average water depth, the channel is being filled from $2000 \mathrm{ml}$ to $14000 \mathrm{ml}$, under different powers provided to the system and different angles of tilt. After recording the figures, we followed the definition of Greenspan, by distinguishing between each mode of the motion, for instance at the lower power provided to the channel the motion appears as closed circles which is considered as laminar flow phase, increasing the power will lead to other phase that may include waves (the flow can be laminar or turbulent) or shock, at this stage in changing the phase Reynolds and Strouhal numbers were computed accordingly, and this point of changing the flow phase is assumed the critical point or the threshold, and then a linear fit regression is proposed. According to this each tilt and each volume will have different critical threshold for the instability observations. The critical Reynolds and Strouhal numbers are computed as:

$$
R_{e}=\frac{\omega R_{h}^{2}}{v}, S_{t}=\frac{\omega R_{h}}{\sqrt{g \bar{h}}} .
$$

Where $\omega$ the frequency of oscillation, $R_{h}$ the hydraulic radius $R_{h}=\frac{A}{P}$, where $A$ the cross-section area which is rectangular one $A=r \cdot \bar{h}, P=r+2 \bar{h}$ the wetted perimeter, $v$ the kinematic viscosity. A simple linear fit is done between the critical thresholds for both Reynolds and Strouhal numbers based on the experimental data is plotted in Figure 5.

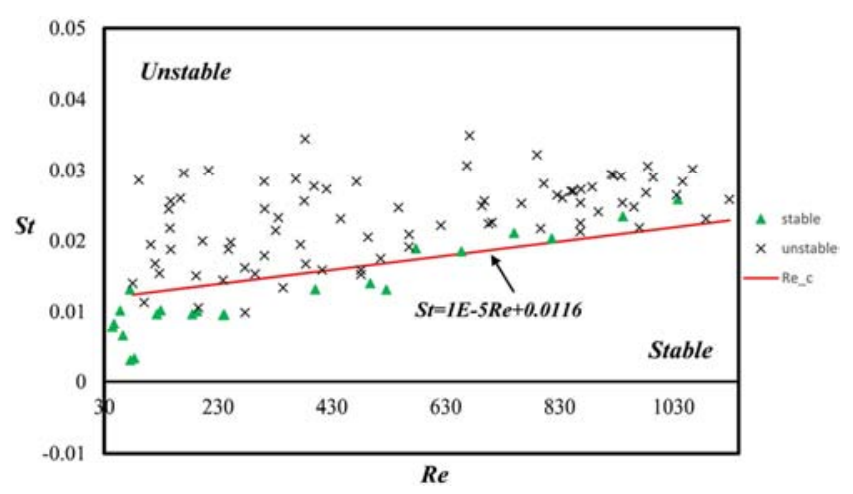

Figure 5. (-) Linear fit for the critical Reynolds number from experimental data, (4) stable experiments, ( $x$ ) unstable experiments.

\section{Weakly Nonlinear Solution}

As stated that those instability features are signs of resonance which is now cannot be predicted theoretically using the linear Eq. (7), and the nonlinear effect should be included in addition to the viscous effects if possible, however here we focus on the nonlinear effect only, precisely the weakly nonlinear case where the scaling of potential Bernoulli Eq. (2) and Laplace Eq. (1) in terms of the shallowness and the amplitude parameters will give finally a new version of $\mathrm{KdV}$ equation that includes the effect of rotation in its coefficients, and it has forcing term that includes the tilt, the full explanation on the mathematical derivation can be found in the previous work [16].

$$
\begin{gathered}
\frac{1}{\varepsilon H}\left(\frac{\lambda^{3} \Omega}{2 c r^{2}}-\frac{c \lambda^{3}}{6 \Omega r^{4}}\right) \eta_{\theta \theta \theta}+\frac{3 c \lambda}{\Omega(\varepsilon H r)^{2}} \eta \eta_{\theta}+\frac{2 \lambda}{\varepsilon^{2} c H} \eta_{t}+ \\
\frac{\tau \Omega r}{\varepsilon \delta c} \sin (\theta-\Omega t)=0 .
\end{gathered}
$$

Where $\varepsilon=\frac{A}{H}$, the amplitude parameter, $A$ the amplitude, $H=\bar{h}$ the average depth of water, $\lambda$ the wavelength, $r=r_{\text {max }}, \quad c=\sqrt{g H}$ the long wave speed, $\delta=\frac{H}{\lambda}$ the shallowness parameter. The solution is compared with the single Kelvin mode that is extracted experimentally in Figure 5 with pretty good match.

\section{Mean Flow}

Many theories on instability features observed in the previous section argued that the instability is related to the azimuthal flow, that is why Lagrangian velocity (drift velocity) measurements using small floating particles to quantify the primary flow were carried out under bore conditions, these non-invasive measurements provide an estimate for the classical mean velocity in the channel. A bilinear regression on the preliminary bore propagation data is depicted in Figure 7, based on Eq. (10). 
$\Omega-\Omega_{\text {drift }}=0.577-12.34 \tau+33.52 H$.

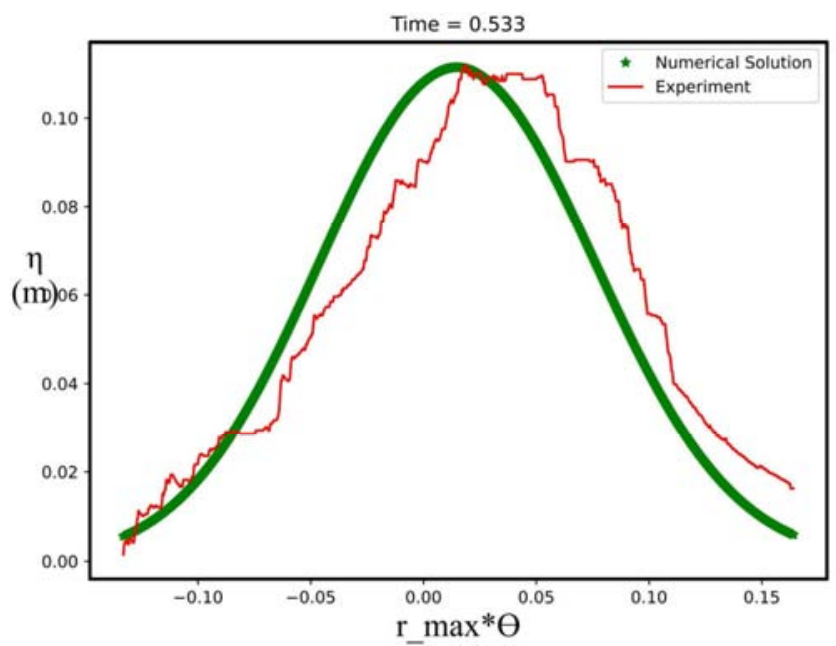

Figure 6. Single Kelvin mode, the analytical solution extracted from Eq. 9. Volume $10000 \mathrm{ml}, \Omega=6.84 \mathrm{rad} / \mathrm{s}, \tau=0.01667$.

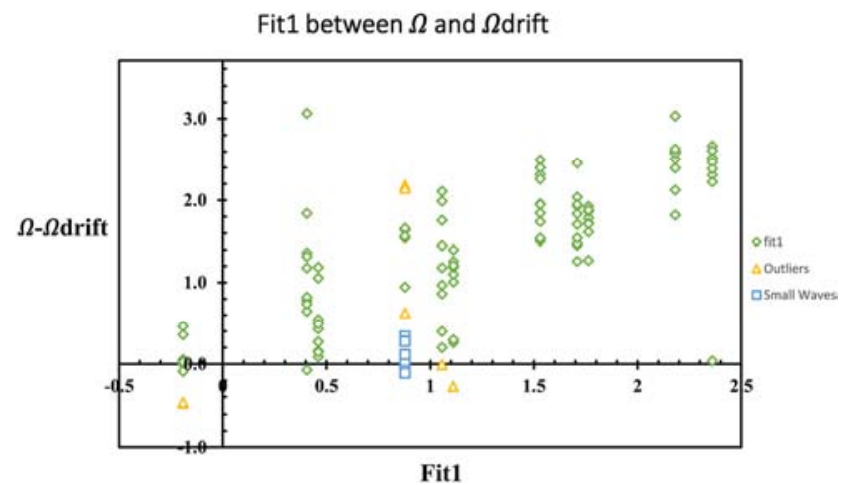

Figure 7. $(\diamond)$, Linear Regression between the actual and the predicted values using Eq. 10, $\Omega-\Omega_{\text {drift }}$, (口) small ripples.

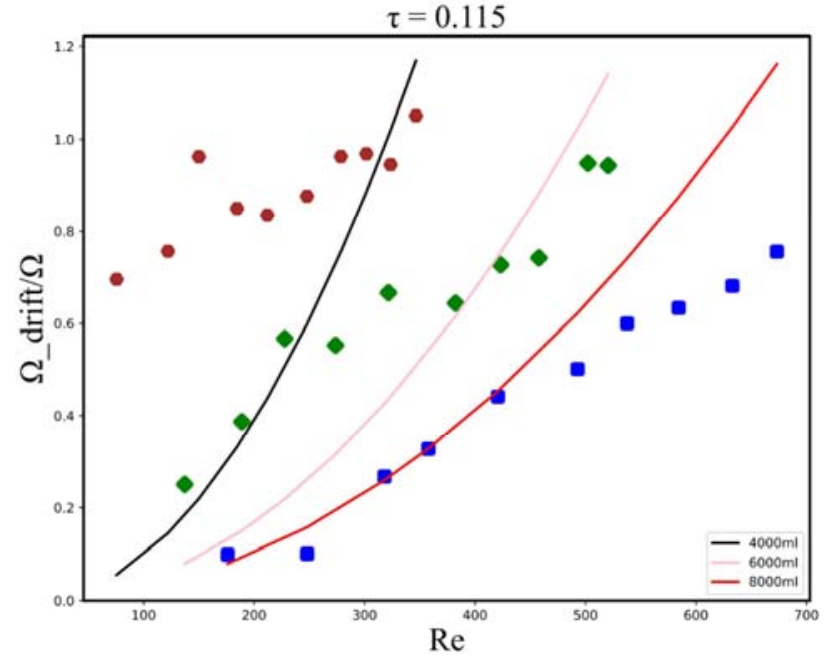

Figure 8. Azimuthal circulation $\Omega_{\text {drift }}$ the solid line is square of mean velocity $U=\Omega r_{\max }$.

Equation (10) states that directly proportional relationship exists between the mean flow and the angle of tilt and rotation rate, and inversely relationship between the mean flow and the average depth of water, this means that the tilt and rotation rate play important role in increasing the instability in the system at early stage of motion, conversely the water volume reduces reaching such level. This result pours in favor of the extracted results in the previous work [3], where the measurements using Acoustic Doppler Velocimeter were carried out directly. The measurement of the azimuthal flow is essential to figure out its effect on wave breaking especially in resonance cases when higher amplitudes appear, as Gunn \& Aldridge [16] for instance stated where they investigated the effect of the mean flow on the resonant collapse problem, they showed that the change in the eigenfrequencies due to nonuniform flow are sufficient to account for the resonant collapse phenomenon. The feature of the azimuthal flow is kind of circulations, the observer of the floated particles sees that their paths of motion are diverted radially. This is another indicator to the secondary motion which is measured previously by McEwan [4], who suggested that these circulations are kind of secondary motion, that is initially created at the expense of the primary rotational energy. A comparison between the drift velocity and the solid body one is plotted against Reynolds number in Figure 8, the solid line is the square of solid body rotational speed.

\section{Conclusion}

In this letter we presented part of the theory that is proposed for studying inertial waves in open cylindrical channel under precession conditions, it mainly depends on the irrotational inviscid assumptions, the work can be found in precise discussion and details of computation in $[3,15]$ here we continued presenting the rest of the results, that accord with real experimental observations and data for the velocity field and wave modes. The instability of the single kelvin mode is discussed, and new instability diagram in terms of Strouhal and Reynolds numbers is presented. The nonlinear part of the problem led to new version of $\mathrm{KdV}$ equation that has forcing term includes the tilt effect, and coefficients that include the rotation effect, it was solved numerically and compared with the experiment with good match. The measurements of the drift velocity reflect the nonuniformity of the base flow, which is suggested to be the mechanism of resonance collapse problem, that is why the measurements studied its effect in terms of the three control parameters of the problem, which are the tilt angle, the rotation rate and the average depth of water, the results showed that the tilt and rotation play drastic role in increasing the instability features in the channel, while increasing the depth reduces reaching such cases, where more energy is needed to trigger the flow.

\section{References}

[1] P. G. Baines. Forced oscillations of an enclosed rotating fluid, J. Fluid Mech. 30 (1967) 533-546.

[2] W. Thomson. Vibration of a Columnar Vortex, Proceedings of the Royal Society of Edinburgh, 5 (1880) 155-168. 
[3] H. E. Alshoufi. On the forced oscillations in a precessing open cylindrical channel, AIP Advances. 11 (2021) 3-23. (a)

[4] A. D. McEwan. Inertial oscillations in a rotating fluid cylinder, J. Fluid Mechanics. 40 (1970) 603-640.

[5] L. Romain, M. Patrice, N. François, and E. Christophe. Precessional instability of a fluid cylinder, J. Fluid Mech. 666 (2011) 104-145.

[6] D. Cébron, M. Le Bars, P. Maubert, and P. Le Gal. Magnetohydrodynamic simulations of elliptical instability in inertial ellipsoids, Geophysical and Astrophysical Fluid Dynamics. 106 (2006) 524-546.

[7] W. V. R. Malkus Precession of the Earth as the Cause of Geomagnetism, Science. 160 (1968) 259-264.

[8] R. Manasseh. Breakdown regimes of inertia waves in a precessing cylinder, J. Fluid Mechanics. 243 (1992) 261-296.

[9] B. N. Agrawal. Dynamic Characteristics of Liquid Motion in Partially Filled Tanks of a Spinning Spacecraft, Journal of Guidance, Control, and Dynamics. 16 (1993) 636-640.

[10] H. R., Vaughn, W. L. Oberkampf, and W. P. Wolfe. Fluid motion inside a spinning nutation cylinder, J. Fluid Mech. 150 (1985) 121-138.

[11] R. R. Kerswell. Secondary instabilities in rapidly rotating fluids: inertial wave breakdown, J. Fluid Mechanics. 382 (1999) 283-306.

[12] R. Thompson. Diurnal tides and shear instabilities in a rotating cylinder, J. Fluid Mechanics, 40 (1970) 737-751.

[13] S. Stergiopoulos, and K. D. Aldridge. Inertial Waves in a Fluid Partially Filling a Cylindrical Cavity during Spin-Up from Rest, Geophys. Astrophys. Fluid Dynamics. 21 (1982) 89-112.

[14] C. Eloy, P. Le Gal, and S. Le Dizès. Elliptic and triangular instabilities in rotating cylinders, J. Fluid Mech. 476 (2003) 357-388.

[15] H. Alshoufi. KdV Equation Model in Open Cylindrical Channel under Precession, Journal of Nonlinear Mathematical Physics. 2021. (b)

[16] J. S. Gunn, and K. D. Aldridge. Inertial wave eigenfrequencies for a nonuniformly rotating fluid, Physics of Fluids. 2 (1990) 2055-2060. 